\title{
Ultrasensitive in-vitro Detection Assay for Non-invasive Alzheimer's Disease Diagnosis
}

\author{
See-Lok Ho, ${ }^{a}$ Hei-Nga Chan, ${ }^{a}$ Hung-Wing Li, ${ }^{*, b}$ and Man-Shing Wong ${ }^{*, b}$ \\ ${ }^{a}$ MIND and Tech, Ltd., Fo Tan, Hong Kong, China \\ ${ }^{b}$ Hong Kong Baptist University, Department of Chemistry, Kowloon Tong, Hong Kong, China \\ Email: hwli@hkbu.edu.hk (H. W. L.), mswong@hkbu.edu.hk (M. S. W.)
}

\begin{abstract}
Alzheimer's disease (AD) is the most prevalent cause of dementia. According to the Alzheimer's Disease Association, there are over 37 million patients currently suffering from AD. To date, there is still no standard method for accurate $A D$ diagnosis. Detection of biomarkers has become a new trend for disease diagnosis as research has shown that alternation of the expression profile of biomarkers occurs over 10 years before the development of any symptoms. Recently, there is a considerable development in the field of ultrasensitive detection assay for AD diagnosis. This perspective gives an updated review of the current state of development on body fluid-based detection assay for non-invasive AD diagnosis.
\end{abstract}

Keywords Alzheimer's disease, biomarkers, non-invasive, diagnosis, immunoassay

Alzheimer's disease (AD) is the most prevalent form of dementia accounting for over $70 \%-80 \%$ of the cases and affecting 50 millions of people worldwide. In every 3 seconds, there is one person diagnosed with dementia. As the aging population is increasing, dementia has become a tremendous socioeconomic issue. In addition, the life expectancy is expected to improve, and the number of people suffering from $A D$ will continue to reach 130 million by $2050 .^{[1,2]}$ Until now, there is no effective treatment available to halt or reverse the underlying pathology of AD. Therefore, early diagnosis and intervention are the best chance to ameliorate the symptoms and delay the progression of this devastating disease. ${ }^{[3]}$

Currently, no single clinical test can provide a confirmative diagnosis of $A D$. Doctors usually make use of a combination of cognitive tests, family medical history, and neurological examinations to evaluate the mental functions of a patient. Besides, blood tests and MRI brain scans are rendered to eliminate other illnesses. ${ }^{[4]}$ As a result, diagnosis of $A D$ is the one left from exclusion. As Bill Gate has mentioned, "It's hard to overstate how important finding a reliable, affordable, and easy-to-use diagnostic is for stopping Alzheimer's." Therefore, an accurate and reliable standard protocol for early $A D$ diagnosis is urgently needed and technically demanded. In recent decade, researchers evidenced that the content and/or profile of $A D$ associated biomarkers varied from normal range at around 15-30 years before the occurrence of any dementia symptoms. Thus, detection of biomarkers represents an emerging method for $A D$ screening. ${ }^{[1,5]}$

Among the $A D$ biomarkers, amyloid- $\beta(A \beta)$, a $4 \mathrm{kDa}$ peptide formed from the Amyloid Precursor Protein (APP) by the enzymatic action of $\beta$ - and $\gamma$-secretases, is believed to be crucial to $A D$ pathogenesis and thus be investigated the most. Several classical ELISA kits for the quantification of biomarkers in cerebrospinal fluid (CSF) have been approved as IVD (in-vitro diagnostic) kit for diagnostic purpose. Fujirebio launched a solid-phase sandwich immunoassay detection kit with peroxidase as the signal reporter for $A \beta_{1-40}$ and $A \beta_{1-42}$ quantification with a limit of detection (LoD) around $2.8 \mathrm{pg} / \mathrm{mL}$. The assay is proven to be highly specific to target biomarkers and do not cross-react with amyloid precursor protein and $A \beta$ isoforms. ${ }^{[6]}$ IBL International developed an ELISA kit to detect $A \beta_{1-40}$ and $A \beta_{1-42}$, with monoclonal antibody capturing the epitopes 38-42 and 35-40, with a horseradish peroxidase as the signal reporter (Figure 1). The detection assay has achieved a LoD of $104 \mathrm{pg} / \mathrm{mL} .^{[7]}$ Euroimmum developed an ELISA assay with Euroimmum analyzer $1-2 \mathrm{P}$ for the automated quantification of $A \beta_{1-40}$ and $A \beta_{1-42}$. The assay obtained a LoD of $70 \mathrm{pg} / \mathrm{mL}$ with a relatively low sample consumption compared to other commercially available ELISA kit. ${ }^{[9]}$ To improve the accuracy of the diagnosis of $A D$, researchers suggested studying the ratio of $A \beta_{1-42} / A \beta_{1-40}$ rather than the single $A \beta$ species. By detecting the content of biomarkers in CSF, these detection approaches have a high diagnostic accuracy towards AD diagnosis with a sensitivity and specificity of over $85 \% .^{[9,10]}$ However, the collection of CSF by means of lumbar punctures is invasive and will cause discomfort and side-effect to the patients.

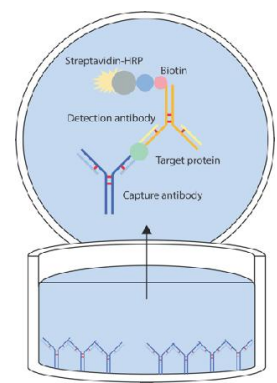

Figure 1 Schematic illustration of ELISA detection approach. Technique relying on the interaction between the target protein and a solid support that is coated with an antibody specific to the target.

It is well known that disease associated biomarkers often circulate throughout the body and exist in different body fluids. The expression profile of circulating $A D$ biomarkers often reflects the pathology and progression and of $A D$. Given that the collection of body fluids, such as blood, urine and saliva, is relatively simple and non-invasive as compared to the 


\section{Perspective}

collection of CSF. Quantifying the content of circulating biomarkers in body fluids has recently drawn tremendous attention as the potential direction of $A D$ diagnosis. Unfortunately, most of the current commercially available ELISA kits cannot satisfy to directly detect such subtle changes of the biomarkers in the complex body fluid matrix.

Quanterix launched a digital highly sensitive immunoassay platform (Simoa) for single molecule detection of $A D$ associated biomarkers. This detection assay is capable of quantifying extremely low amount of biomarkers in most of the body fluids. With its high sensitivity and specificity, this detection platform is suitable for detection of trace amount of $A \beta$ peptides in plasma sample. ${ }^{[11]}$ Unfortunately, the price of this automated detection instrument is very high, which might not be affordable for general research laboratory and testing laboratory. Chan and coworkers has previously reported an ultra-sensitive and more cost-effective alternative for the detection of AD biomarkers in body fluids. The detection approach features the usage of a magnetic detection probe to capture the target protein (Figure 2). The immunocomposite is fluorescently labelled by a specific turn-on fluorophore. Unlike other commercially available fluorophores, this tailor-made fluorophore shows a remarkably high fluorescence enhancement upon binding to proteins that greatly amplifies the read-out signal of the assay. Hence, the sensitivity of the detection assay (LoD) can be lowered down to femto $(10-15)$ molar regime $(0.1 \mathrm{pg} / \mathrm{mL}){ }^{\left[{ }^{[9]}\right.}$ Therefore, it is sensitive enough to directly measure the content of such proteins in saliva or urine. It has a wide dynamic range and only requires a minute amount of sample (a few microliter). This assay can be practically useful as a rapid and low-cost alternative for accurate diagnosis of $A D$. To improve the robustness and versatility of the immuno-magnetic nano-platform further, Chan and coworkers have recently developed an ultrasensitive multiplex assay based on a hybrid antibody-aptamer sandwich immuno- complexes for $A \beta_{1-42}$ and tau proteins detection. The use of aptamers offers many practical advantages over antibodies, which includes higher or comparable target affinity, higher stability, and lower batch-to-batch variation. This newly developed assay is shown to have a higher sensitivity with one order of magnitude lower in LoD (4-8 fM (38-165 fg/mL)). ${ }^{[12]}$
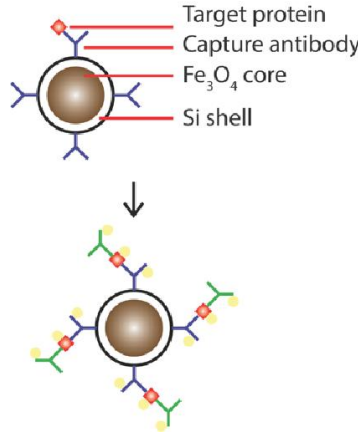

Figure 2 Schematic illustration of immuno-magnetic detection platform. The detection assay is mainly based highly specific lock and key interaction between antibody and protein.
Meanwhile, development of sensitive platform for other AD biomarkers is also actively pursued. The success of such a development will facilitate early diagnosis of $A D$, as it is of paramount importance for the aspect of preventive strategies. Despite recent advances in developing analytical approaches and tools for $A D$ biomarkers detection, applying them reliably and reproducibly as a clinical routine for $A D$ diagnosis still remains challenging. We hope a widespread progress in clinical and hospital usage will be seen in the near future.

\section{References}

[1] Scheltens, P.; Blennow, K.; Breteler, M. M. B.; de Strooper, B.; Frisoni, G. B.; Salloway, S.; Van der Flier, W. M. Lancet 2016, 388, 505

[2] Gaugler, J.; James, B.; Johnson, T.; Marin, A.; Weuve, J.; Alzheimer's, A. Alzheimers Dement. 2019, 15, 321.

[3] Hodson, R. Nature 2018, 559, S1.

[4] Zhou, T.; Thung, K. H.; Zhu, X. F.; Shen, D. G. Hum. Brain Mapp. 2019, 40, 1001.

[5] Olsson, B.; Lautner, R.; Andreasson, U.; Ohrfelt, A.; Portelius, E.; Bjerke, M.; Holtta, M.; Rosen, C.; Olsson, C.; Strobel, G.; Wu, E.; Dakin, K.; Petzold, M.; Blennow, K.; Zetterberg, H. Lancet Neurol. 2016, 15,673

[6] Vanderstichele, H.; Van Kerschaver, E.; Hesse, C.; Davidsson, P.; Buyse, M. A.; Andreasen, N.; Minthon, L.; Wallin, A.; Blennow, K.; Vanmechelen, E. Amyloid 2000, 7, 245.

[7] Lewczuk, P.; Lelental, N.; Spitzer, P.; Maler, J. M.; Kornhuber, J. J. Alzheimers Dis. 2015, 43, 183.

[8] Chiasserini, D.; Biscetti, L.; Farotti, L.; Eusebi, P.; Salvadori, N.; Lisetti, V.; Baschieri, F.; Chipi, E.; Frattini, G.; Stoops, E.; Vanderstichele, H.; Calabresi, P.; Parnetti, L. J. Alzheimers Dis. 2016, 54, 55

[9] Chan, H. N.; Xu, D.; Ho, S. L.; Wong, M. S.; Li, H. W. Chem. Sci. 2017, 8, 4012

[10] Molinuevoa, J. L.; Gispert, J. D.; Dubois, B.; Heneka, M. T.; Lleo, A.; Engelborghs, S.; Pujol, J.; de Souza, L. C.; Alcolea, D.; Jessen, F.; Sarazin, M.; Lamari, F.; Balasa, M.; Antonell, A.; Rami, L. J. Alzheimers Dis. 2013, 36, 67.

[11] Wilson, D. H.; Rissin, D. M.; Kan, C. W.; Fournier, D. R.; Piech, T.; Campbell, T. G.; Meyer, R. E.; Fishburn, M. W.; Cabrera, C.; Patel, P. P.; Frew, E.; Chen, Y.; Chang, L.; Ferrell, E. P.; von Einem, V.; McGuigan, W.; Reinhardt, M.; Sayer, H.; Vielsack, C.; Duffy, D. C. Jala 2016, 21, 533

[12] Chan, H. N.; Xu, D.; Ho, S. L.; Wong, M. S.; Li, H. W. Theranostics 2019, 9, 2939 